\title{
Landing site positioning and descent trajectory reconstruction of Tianwen-1 on Mars
}

\author{
Anran Wang, Li Wang(凶), Yinuo Zhang, Baocheng Hua, Tao Li, Yang Liu, and Dayong Lin
}

Beijing Institute of Control Engineering, Beijing 100190, China

\begin{abstract}
Tianwen-1 (TW-1) is the first Chinese interplanetary mission to have accomplished

orbiting, landing, and patrolling in a single exploration of Mars. After safe landing, it is essential to reconstruct the descent trajectory and determine the landing site of the lander. For this purpose, we processed descent images of the TW-1 optical obstacle-avoidance sensor (OOAS) and digital orthophoto map (DOM) of the landing area using our proposed hybrid-matching method, in which the landing process is divided into two parts. In the first, crater matching is used to obtain the geometric transformations between the OOAS images and DOM to calculate the position of the lander. In the second, feature matching is applied to compute the position of the lander. We calculated the landing site of TW-1 to be $109.9259^{\circ} \mathrm{E}, 25.0659^{\circ} \mathrm{N}$ with a positional accuracy of $1.56 \mathrm{~m}$ and reconstructed the landing trajectory with a horizontal root mean squared error of $1.79 \mathrm{~m}$. These results will facilitate the analyses of the obstacle-avoidance system and optimize the control strategy in the follow-up planetary-exploration missions.
\end{abstract} KEYWORDS

\author{
Tianwen-1 (TW-1) \\ descent trajectory \\ landing site \\ hybrid matching \\ crater matching \\ feature matching
}

\section{Research Article \\ Received: 16 September 2021 \\ Accepted: 25 October 2021 \\ (C) The Author(s) 2022}

\section{Introduction}

Tianwen-1 (TW-1) is the first Chinese Mars-exploration mission to have realized orbiting, landing, and patrolling simultaneously. Its success of TW-1 made China the third country after the former Soviet Union and the United States to achieve a Mars-landing exploration [1-3]. The probe was launched on July 23, 2020 and the spacecraft was captured by Mars on February 10, 2021 after seven months of transit. For the next three months, the probe conducted reconnaissance for choosing the target landing site. On May 15, 2021, the lander separated from the orbiter and landed in the preselected landing zone in the southern part of the Martian Utopian Plain. Landing process is the most critical aspect of a Mars exploration. For a better understanding of the autonomous-control results and analysis of the obstacle-avoidance strategy used, it is essential to reconstruct the descent trajectory and locate the landing site of lander after safe landing.

Several studies have focused on the descent trajectory reconstruction and landing site positioning of lander. For example, radio measurements [4-6], including radio ranging and very long baseline interferometry (VLBI), have been widely used to reconstruct landing trajectories. However, owing to the complexity and uncertainty of the Martian environment, the positioning accuracy with this method is only of the order of few kilometers. Moreover, this method cannot be applied without Earth-based radio tracking or telemetry data. Therefore, an image-based method was developed that utilizes images captured by the onboard camera along with known georeferenced data of the planetary surface. This method enables more precise calculations of the landing site and descent trajectory and is not dependent on particular exploration environments. For the Chang'e-3 (CE-3) and Chang'e-4 (CE-4) missions, a landing camera with a high frame rate of 10 frames/s, which obtained thousands of continuous images, was employed during the entire descent process with an image overlap rate of more than $94 \%$. A number of image-based methods have been proposed to make use of these images. For instance, using the photogrammetric method, Liu et al. [7] selected 180 images in $1 \mathrm{~s}$ intervals from the landing images and used CE2TMap2015 as the 
georeferenced data for reconstructing the powered-descent trajectory. This method needs to automatically extract and match a large number of feature points throughout the overlaps of the landing images. In addition, the matched points could be input into the photogrammetric bundle adjustment to reconstruct the descent trajectory of the CE-4 lander. Taking engineering requirements into account, Wang et al. [8] determined the landing point of the CE-4 lander using high-precision image-matching and geometric-transformation methods. The landing point was initially located with near-real-time high-compression-ratio descent sequence images. Then, the estimate was refined using the replayed low-compression-ratio descent images. This method can calculate the landing point fast. In addition, in Ref. [9], control points were acquired by matching the descent images with the digital orthophoto map (DOM) generated from CE-2 images, and the descent trajectory of the CE-3 lander was obtained using the SIFT feature-matching and bundle-adjustment methods.

In this study, an image-based method was employed to fulfill the precise positioning requirements of TW-1. However, the limited transmission rate and high time interval for transmission between the Earth and Mars makes it unrealistic to download the data obtained by the landing camera. Consequently, OOAS image data were used in this study. The optical obstacle-avoidance sensor (OOAS) is one of the payloads installed on the TW-1 lander, which recorded the entire landing process in a small number of images. Note that there are two disadvantages to using OOAS images. First, the images were downloaded with compression, leading to loss of information. Second, the high sun-elevation angle during the landing of TW-1 resulted in low image contrast and poor image quality.

Considering the properties of the OOAS images, we propose a hybrid-matching approach which executes descent-trajectory reconstruction in two parts. First, for the case of the lander at a high altitude, the crater is clearly recognizable in the OOAS images, which enables us to employ the crater-matching method. In contrast, in the case of the lander at a low altitude, the number of craters in the OOAS images is limited, and the feature-matching method needs to be applied. Based on hybrid-matching method, the landing site and trajectory were determined and reconstructed smoothly, which does not rely on Earth-based telemetry data and multi-landing images. In conclusion, for the first time, we located the landing site of $\mathrm{TW}-1$ as $109.9259^{\circ} \mathrm{E}, 25.0659^{\circ} \mathrm{N}$ with an accuracy of $1.56 \mathrm{~m}$ and reconstructed the landing trajectory with a horizontal root mean squared (RMS) error of $1.79 \mathrm{~m}$. These results can aid the analysis of the obstacle-avoidance system and control-strategy optimization for the follow-up planetary-exploration missions.

The rest of this paper is organized as follows. In Section 2, we describe the dataset including the OOAS images and DOM data and define the coordinate systems. In Section 3, the technical details of the hybrid-matching method and related experimental processes are presented. Sections 4 and 5 present the procedures and results, respectively, of the landing site determination and landing trajectory reconstruction. Finally, a summary and conclusion are presented in Section 6.

\section{Dataset description and coordinate- system definition}

\subsection{Dataset description}

To reconstruct the landing trajectory and determine the landing site, we used the images captured by the onboard OOAS and high-resolution DOM data of the Martian surface. The position of the lander at the time of OOAS image capture was calculated by detecting corresponding features between the OOAS image and DOM data. The details of the two datasets are presented in the following.

\subsubsection{OOAS images}

The OOAS is one of the payloads on the TW-1 lander responsible for ensuring safe landing. The OOAS records the entire powered descent starting from when the lander releases the heat shield (approximately $10 \mathrm{~km}$ from the Martian surface). The frame rate of OOAS was approximately 1 frame per $4 \mathrm{~s}$, and its main parameters are listed in Table 1. The OOAS images contain position information for TW-1, which can be used to locate the landing site and reconstruct the descent trajectory. In this study, 36 OOAS images were selected for use in landing-trajectory reconstruction, as shown in the Appendix. The capture time of the image is presented in Fig. 1: The capture time of the first image is the origin, and the horizontal and vertical axes represent OOAS images 1-36 and their capture time, respectively.

\subsubsection{DOM data}

The DOM data were used as geographical reference data 
Table 1 Main parameters of OOAS

\begin{tabular}{cc}
\hline Performance parameter & Value \\
\hline Field of view $\left(^{\circ}\right)$ & $30 \times 30$ \\
Focal length $(\mathrm{mm})$ & 19.94 \\
Effective pixel number & $2048 \times 2048$ \\
Pixel size on focal plane $(\mu \mathrm{m})$ & 5.5 \\
Data-compression ratio & $32: 1$ \\
Principal point (pixel) & $(1026.4,1029.7)$ \\
\hline
\end{tabular}

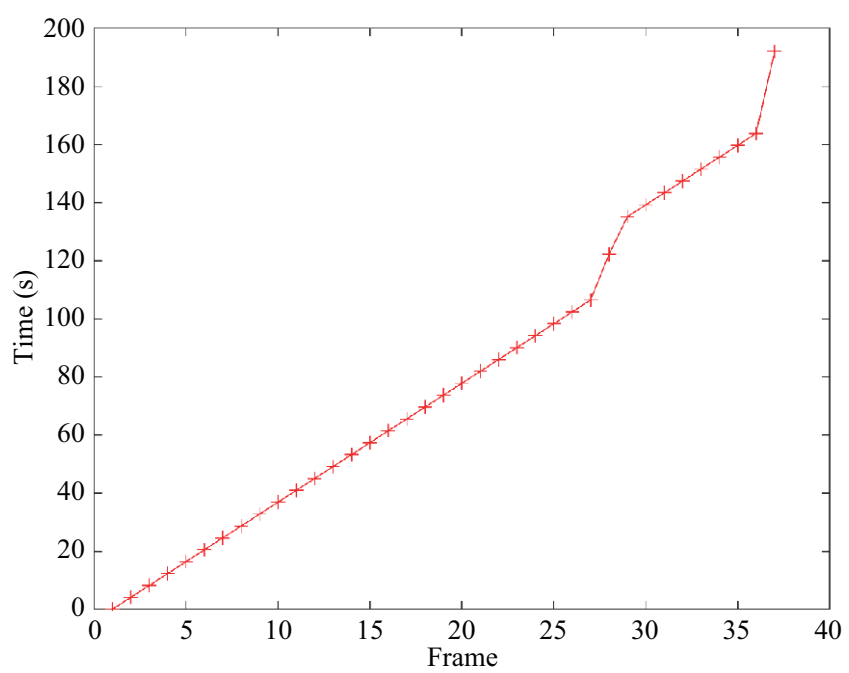

Fig. 1 Time of capture of OOAS images.

for landing site positioning and trajectory reconstruction. In this study, the DOM data (shown in Fig. 2) of the landing zone were generated from the images captured by the onboard high-resolution camera [10,11]. The DOM data cover the landing area from $109^{\circ} 48^{\prime} \mathrm{E}$ to $109^{\circ} 58^{\prime} \mathrm{E}$ and from $24^{\circ} 58^{\prime} \mathrm{N}$ to $25^{\circ} 6^{\prime} \mathrm{N}$ with a spatial resolution of $0.7 \mathrm{~m}$, which is available at the Data Publishing and Information Service System of China's Lunar Exploration Program [12].

\subsection{Coordinate-system definition}

For trajectory reconstruction, three coordinate systems need to be defined: a georeferenced coordinate system $\boldsymbol{X}_{\mathrm{m}}$, an image coordinate system $\boldsymbol{X}_{\mathrm{i}}$, and a camera coordinate system $\boldsymbol{X}_{\mathrm{c}}$. These were defined as follows (Fig. 3). First, the origin of $\boldsymbol{X}_{\mathrm{m}}$ was placed at the center of the map, and the axes $x_{\mathrm{m}}, y_{\mathrm{m}}$, and $z_{\mathrm{m}}$ defined as the east, north, and vertical directions, respectively. Second, $\boldsymbol{X}_{\mathrm{i}}$ was defined as a $2 \mathrm{D}$ coordinate system with the $x_{\mathrm{i}}$ and $y_{\mathrm{i}}$ axes parallel to the column and row of the OOAS image, respectively. Finally, for $\boldsymbol{X}_{\mathrm{c}}$, the origin was placed at the optical center of the camera with the axes $x_{\mathrm{c}}$ and $y_{\mathrm{c}}$ pointing in the same directions as those in $\boldsymbol{X}_{\mathrm{i}}$. The

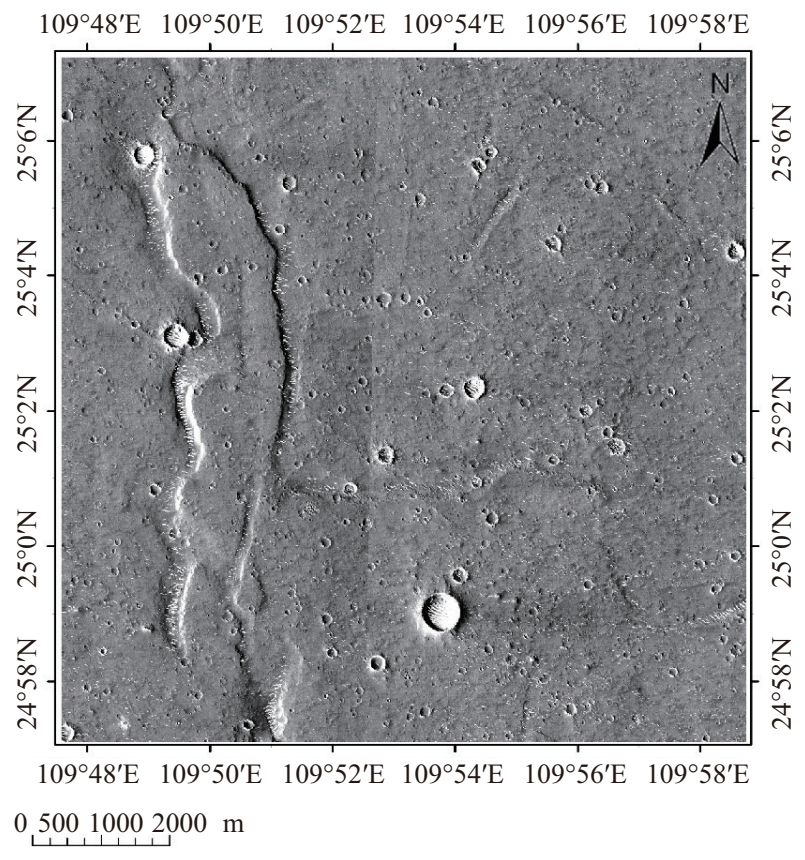

Fig. 2 DOM data of the landing area.

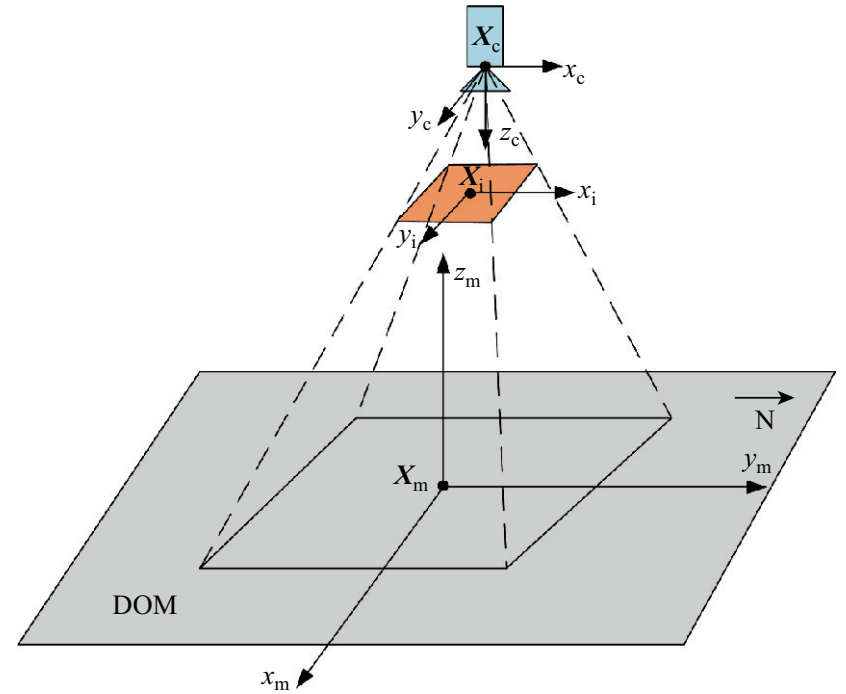

Fig. 3 Coordinate-system definition.

$z_{\mathrm{c}}$ axis points along the main optical axis and forms a right-handed set with $x_{\mathrm{c}}$ and $y_{\mathrm{c}}$.

\section{$3 \quad$ Hybrid-matching method}

Descent trajectory and landing site calculation requires a geometric transformation $\boldsymbol{H}_{n \mathrm{D}}$ between the lander and Martian surface, where $\boldsymbol{H}_{n \mathrm{D}}$ is computed using the matching-point pairs between the OOAS images and DOM data. In this paper, a hybrid-matching method based on crater matching and feature matching is proposed for this, as shown in Fig. 4. Note that 


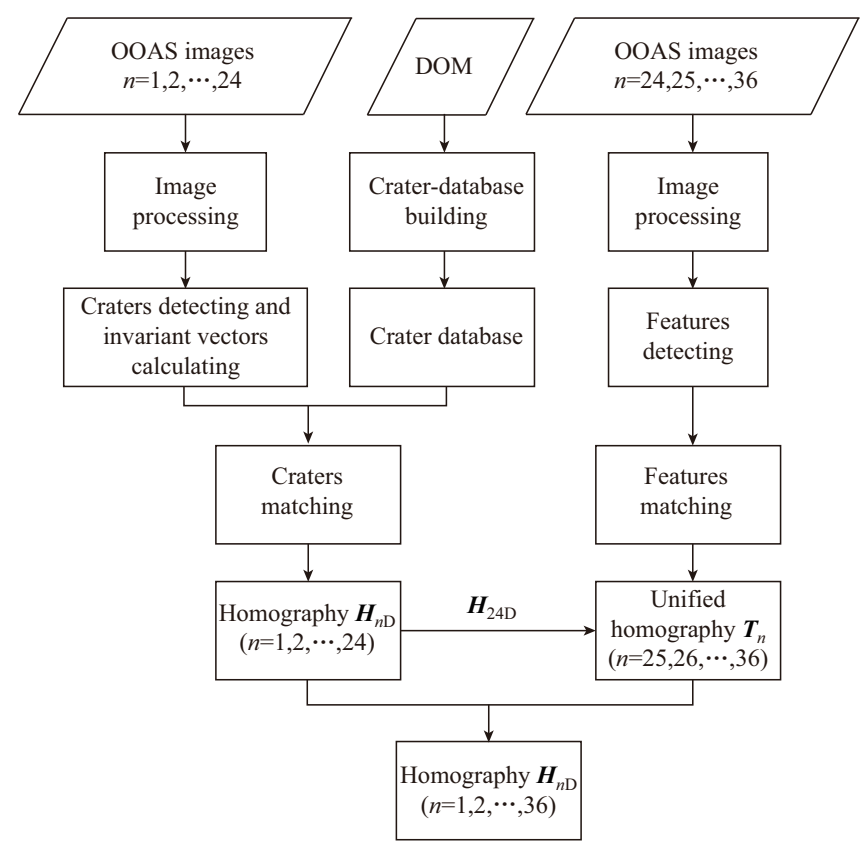

Fig. 4 Data processing flow in hybrid-matching approach.

because of the low contrast and poor quality of the OOAS images, image enhancement was performed prior to the matching process. After image processing, the following procedures were applied to the OOAS images. First, for the lander at a high altitude (images 1-24), the $\boldsymbol{H}_{n \mathrm{D}}(n=1,2, \cdots, 24)$ was estimated by the crater-matching method, which matches the craters detected in the OOAS images with the known crater database built from the DOM data. Subsequently, for the case of the low-altitude lander (images 25-36), the matched craters are insufficient. Thus, in this case, the $\boldsymbol{H}_{n \mathrm{D}}(n=25,26, \cdots, 36)$ was calculated using the feature-matching method as follows. First, we computed the corresponding relationship $\boldsymbol{T}_{n}(n=25,26, \cdots, 36)$ between the OOAS images from the 25th to the 36th and the 24th OOAS image through inter-frame feature matching. With the obtained geometric transformation $\boldsymbol{H}_{24 \mathrm{D}}$ at high altitudes, $\boldsymbol{T}_{n}(n=25,26, \cdots, 36)$ was converted to the georeferenced coordinate system, and therefore, the last part of $\boldsymbol{H}_{n \mathrm{D}}(n=25,26, \cdots, 36)$ was acquired.

Being one of the most common features on the surfaces of extraterrestrial bodies, craters are suitable for use in autonomous navigation and obstacle identification by spacecraft. In the crater-matching process, the corresponding points can be obtained by matching the craters extracted from the OOAS images with those in the pre-established crater database [13-15]. In this study, crater matching was mainly applied to the OOAS images taken at altitudes above $3 \mathrm{~km}$ from the Martian surface, and the matching procedure is outlined in Algorithm 1.

\begin{tabular}{c}
\hline Algorithm $\mathbf{1}$ Process of crater matching \\
\hline Input: OOAS images (1-24), DOM data \\
Output: Geometric transformations \\
$\boldsymbol{H}_{n \mathrm{D}}(n=1,2, \cdots, 24)$ between OOAS \\
images and DOM data
\end{tabular}

Algorithm:

Step 1: Build crater database based on DOM data.

Step 2: Extract craters from the OOAS images and generate the list of crater triangles and triangular invariants.

Step 3: Match the list obtained in Step 2 with the crater database using triangle matching method and determine the crater matches.

Step 4: Compute the geometric transformations $\boldsymbol{H}_{n \mathrm{D}}(n=1,2, \cdots, 24)$ between the OOAS images and DOM using the crater matches from Step 3.

First, the crater database was created based on the DOM data. The main parameters of the crater database are as follows.

(1) Crater locations and diameters of the DOM. In this study, 78 craters with radii in the range of 5.5-175.6 m were selected from the DOM. The distributions of these craters are shown in Fig. 5(a).

(2) List of crater triangles and triangular invariants. Three craters form a crater triangle, and all possible crater triangles were catalogued in a list. Each catalogued crater triangle contains triangular invariants as follows: the smallest, middle, and largest angles; cosine of the largest and smallest angles; three crater diameter ratios; and the sense of rotation for each triangle. In particular, the crater diameter ratios were calculated as the ratio of the crater diameters to the longest leg of the corresponding crater triangle. The sense of rotation of the crater triangle indicates a clockwise or counterclockwise path, which is produced by tracing the triangle from the vertex of its smallest angle to that of the middle angle and then to the largest angle [16]. As an illustration, Fig. 5(b) depicts an image of a typical crater triangle. In Fig. 5(b), the smallest, middle, and largest angles are denoted as $\alpha_{\mathrm{s}}, \alpha_{\mathrm{m}}$, and $\alpha_{\mathrm{l}}$, respectively. The diameters of craters $i, j$, and $k$ were divided by the length of $R_{i j}$ to 


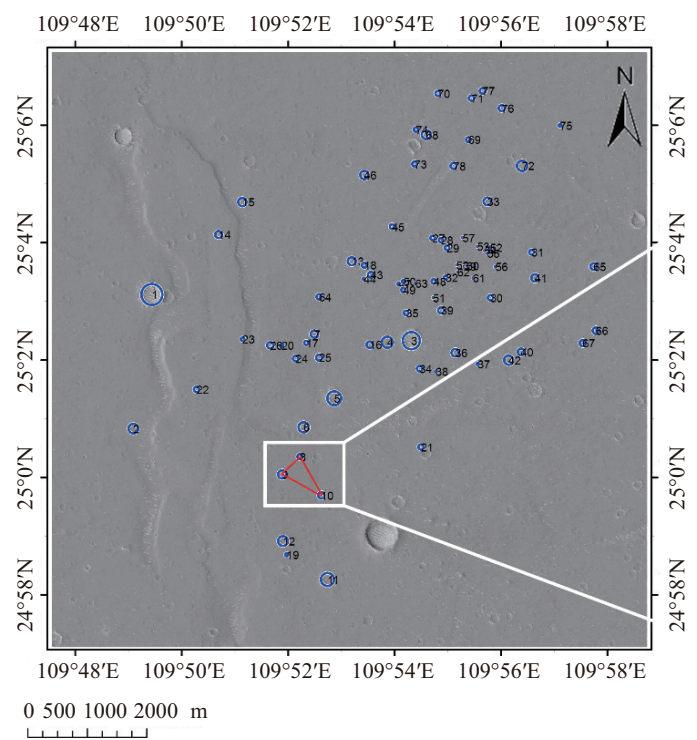

(a) Distribution of craters

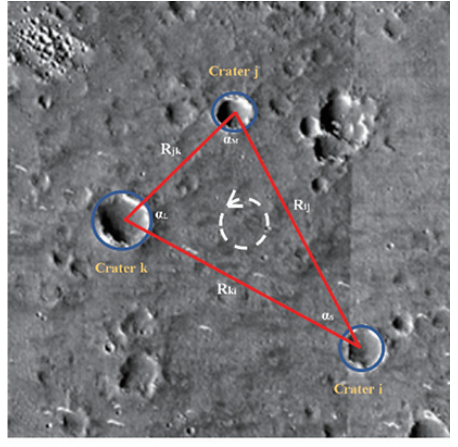

(b) Crater triangle

Fig. 5 Crater distributions in the DOM of the landing area and an example of a crater triangle.

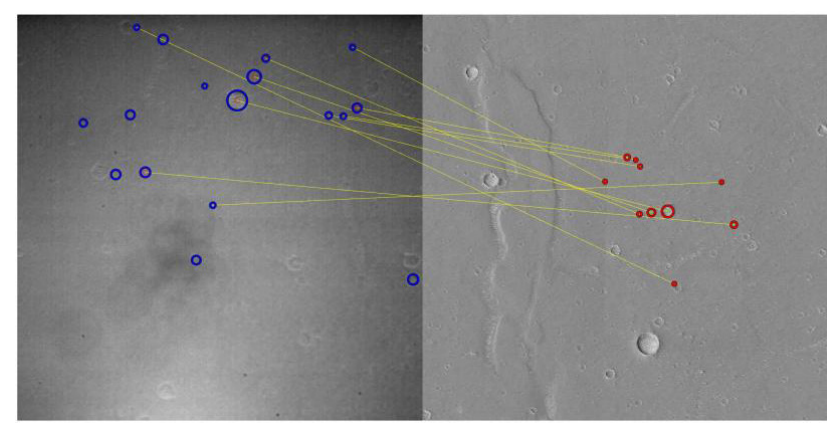

(a) Matching craters between the 1st landing image and DOM

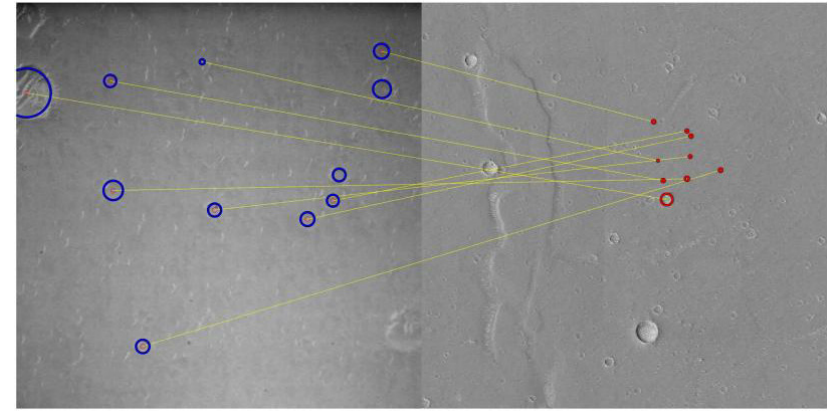

(b) Matching craters between the 24th landing image and DOM

Fig. 6 Matching craters between the 1st and 24th OOAS images and DOM.

obtain the crater diameter ratios. The sense of rotation of the crater triangle in Fig. 5(b) is counterclockwise.

Second, the craters were extracted from the OOAS images, and a list of crater triangles and triangular invariants for the OOAS images was made (the OOAS list of crater triangles and triangular invariants was the same as that of the crater database).

Third, using the triangle-matching method (see Refs. $[16,17]$ for a description of the algorithm), the crater triangle list of OOAS images was matched with that of the crater database. Thus, we obtained the matching crater pairs between the OOAS images and DOM. The matching crater pairs of the 1st and 24th OOAS images are shown in Fig. 6.

Finally, using the centers of the crater matches, we obtained a group of matching point pairs between the image coordinates and georeferenced coordinates. The geometric transformations $\boldsymbol{H}_{n \mathrm{D}}(n=1,2, \cdots, 24)$ between the OOAS images and DOM were computed by matching point pairs [18].

With decreasing spacecraft altitude, crater matching becomes unreliable owing to the decreasing number of matched craters. For the 25th to the 36th images, which were captured below $3 \mathrm{~km}$, the feature-matching method was used to construct the geometric transformations $\boldsymbol{H}_{n \mathrm{D}}(n=25,26, \cdots, 36)$ between the OOAS images and DOM. The feature-matching process is shown in Algorithm 2.

The feature-matching process can be described as follows. First, by selecting and matching the point 

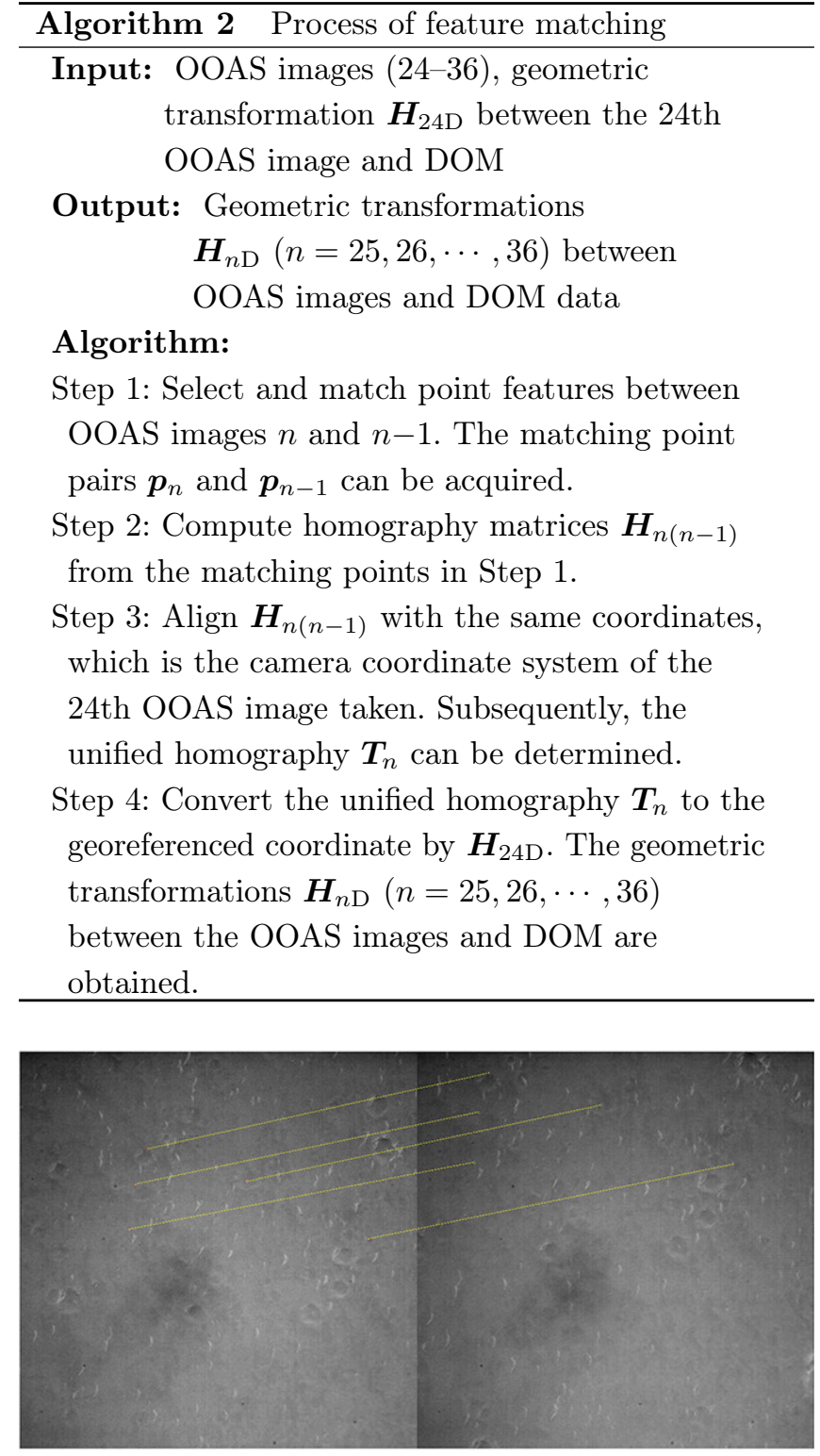

Fig. 7 Feature matching result between OOAS images 24 and 25 .

features [19] from the OOAS images $n$ and $n-1(n=$ $25,26, \cdots, 36)$, the matching point pairs $\boldsymbol{p}_{n}$ and $\boldsymbol{p}_{n-1}$ between the adjacent OOAS images were obtained. The matching points between OOAS images 24 and 25 are shown in Fig. 7.

Second, homography matrices were computed from the matching point pairs. The homogeneous coordinates of $\boldsymbol{p}_{n}$ and $\boldsymbol{p}_{n-1}$ are $\boldsymbol{P}_{n}$ and $\boldsymbol{P}_{n-1}$, respectively. The corresponding relationship is as Eq. (1):

$$
\boldsymbol{P}_{n-1}=\boldsymbol{H}_{n(n-1)} \boldsymbol{P}_{n}
$$

where $\boldsymbol{H}_{n(n-1)}$ can be solved by the least-squares method when there are multiple sets of matching point pairs.

Third, the 24th OOAS image and its camera coordinate system were used as the reference image and reference coordinate system, respectively. The OOAS images can be aligned with the reference image as Eq. (2):

$$
\boldsymbol{T}_{n}=\boldsymbol{H}_{n(n-1)} \boldsymbol{T}_{n-1}(n>24)
$$

where $\boldsymbol{T}_{n}$ is the unified homography between the camera coordinate system of images $25-36$ and the reference coordinate system. With $\boldsymbol{T}_{n}$, we projected the OOAS images (25-36) onto the 24th OOAS image. The projection results are shown in Fig. 8.

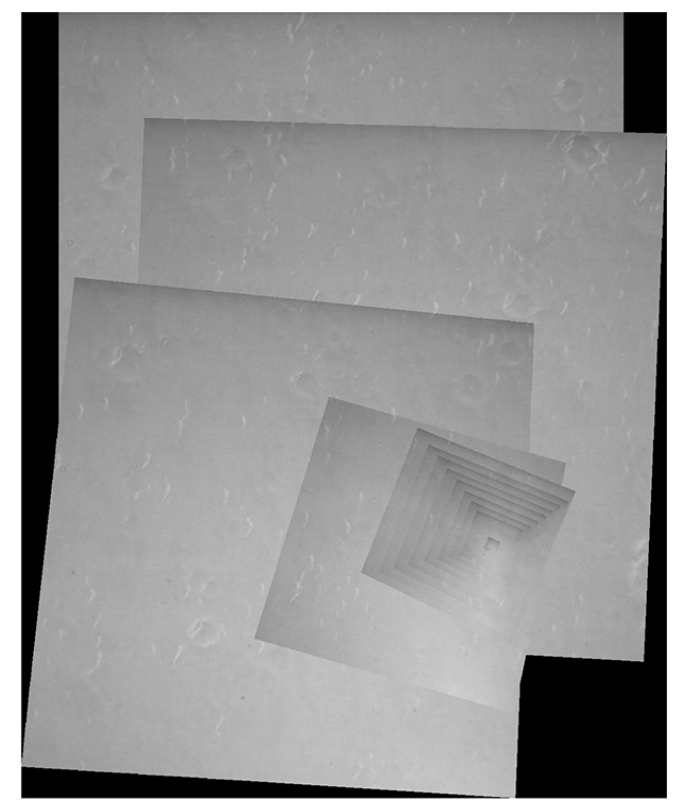

Fig. 8 Projection result of OOAS images 25-36 onto the OOAS image 24 .

Finally, with the geometric transformation between the reference coordinate system and georeferenced coordinate system $\boldsymbol{H}_{24 \mathrm{D}}$, the geometric transformations $\boldsymbol{H}_{n \mathrm{D}}$ between the OOAS images (25-36) and DOM can be acquired by

$$
\boldsymbol{H}_{n \mathrm{D}}=\boldsymbol{T}_{n} \boldsymbol{H}_{24 \mathrm{D}}(n>24)
$$

By combining the geometric transformations obtained by crater matching and feature matching, we obtained all geometric transformations $\boldsymbol{H}_{n \mathrm{D}}(n=1,2, \cdots, 36)$, which were then used in the following landing site positioning and descent trajectory reconstruction processes.

\section{TW-1 landing site positioning}

Because the lander adopted a vertical-descent method for approaching the landing zone during the final period of 
the descent, we set the center of the last OOAS image as the landing site. The OOAS images were projected onto the DOM with the geometric transformations $\boldsymbol{H}_{n \mathrm{D}}(n=$ $1,2, \cdots, 36)$, as shown in Fig. 9, where the center of the last OOAS image is marked with a red cross in DOM. According to the range of DOM, the longitude and latitude of the landing site were confirmed as $109.9259^{\circ}$ E and $25.0659^{\circ} \mathrm{N}$.

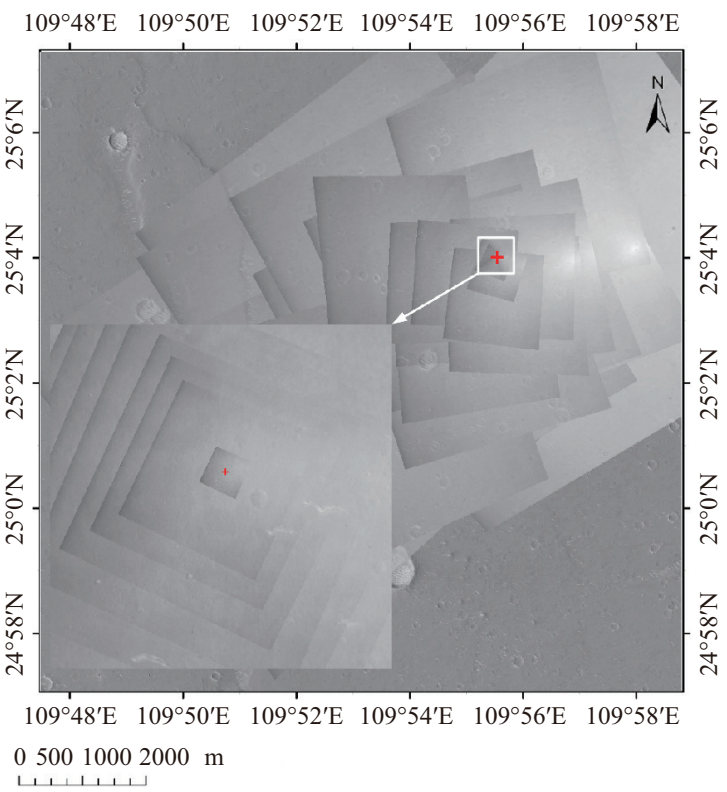

Fig. 9 Projection of OOAS images onto DOM (red cross indicates the landing site).

To verify the accuracy of the landing site location, the result was compared with the location of landing site seen in the high-resolution image from the China National Space Administration [20]. Using the four craters near the landing site as references, the landing site obtained using the proposed method was seen to be consistent with that in the real image, as shown in Fig. 10. Moreover, when the high-resolution image was projected onto the DOM (Fig. 11), the calculated landing point differed by only $1.56 \mathrm{~m}$ from that observed in the photograph.

\section{TW-1 descent trajectory recons- truction}

To evaluate the autonomic-control strategy and obstacle-avoidance method, the landing process of TW-1 was recovered and is described in this section. With the geometric transformation $\boldsymbol{H}_{n \mathrm{D}}=\left[\boldsymbol{h}_{1}, \boldsymbol{h}_{2}, \boldsymbol{h}_{3}\right](n=$ $1,2, \cdots, 36$ ) obtained in Section 3 , the rotation matrix
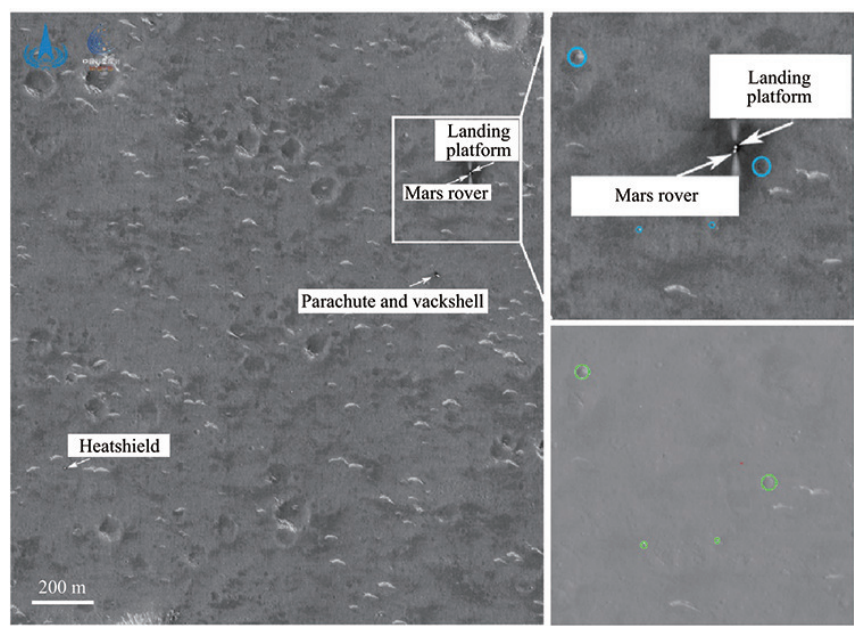

Fig. 10 Landing site comparison (left: high-resolution image of landing site location released by China National Space Administration, top right: zoomed-in view of the landing platform, and bottom right: landing site location determined in this study).

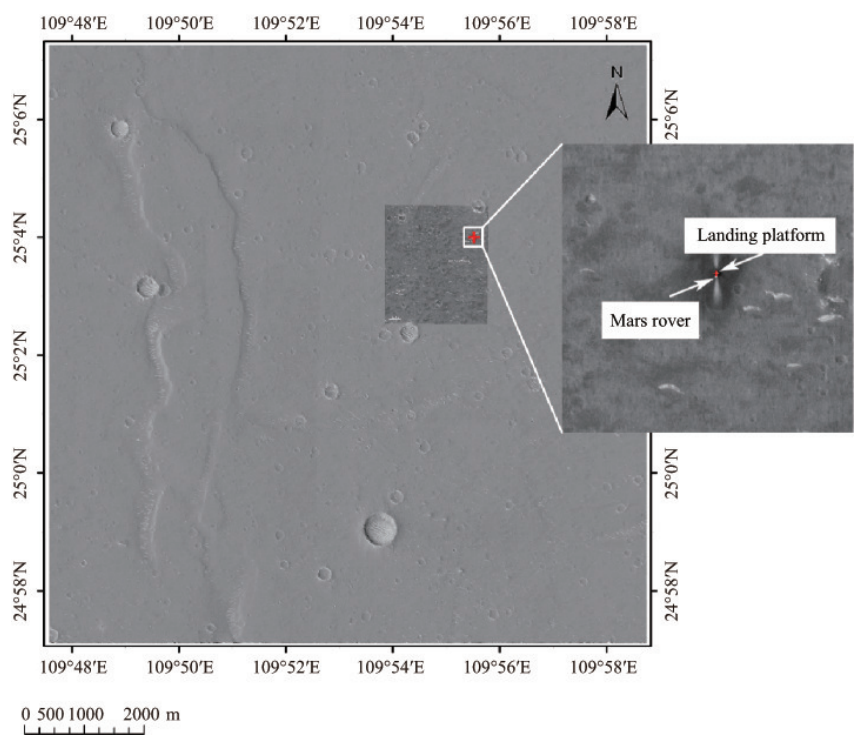

Fig. 11 Projection of high-resolution image onto DOM (red cross marks the analytically determined landing point).

$\boldsymbol{R}=\left[\boldsymbol{r}_{1}, \boldsymbol{r}_{2}, \boldsymbol{r}_{3}\right]$ and position vector $\boldsymbol{t}$ are calculated as

$$
\left\{\begin{array}{l}
\boldsymbol{r}_{1}=z_{\mathrm{c}} \boldsymbol{K}^{-1} \boldsymbol{h}_{1} ; \boldsymbol{r}_{2}=z_{\mathrm{c}} \boldsymbol{K}^{-1} \boldsymbol{h}_{2} ; \boldsymbol{r}_{3}=\boldsymbol{r}_{1} \times \boldsymbol{r}_{2} \\
\boldsymbol{t}=z_{\mathrm{c}} \boldsymbol{K}^{-1} \boldsymbol{h}_{3} \\
z_{\mathrm{c}}=\frac{1}{\left\|\boldsymbol{K}^{-1} \boldsymbol{h}_{1}\right\|}=\frac{1}{\left\|\boldsymbol{K}^{-1} \boldsymbol{h}_{2}\right\|}
\end{array}\right.
$$

where $\boldsymbol{h}_{1}, \boldsymbol{h}_{2}, \boldsymbol{h}_{3}$ and $\boldsymbol{r}_{1}, \boldsymbol{r}_{2}, \boldsymbol{r}_{3}$ are the column vectors of $\boldsymbol{H}_{n \mathrm{D}}$ and $\boldsymbol{R}$, respectively. $\boldsymbol{K}$ is the internal camera parameter matrix, which is obtained from Table $1 . z_{\mathrm{c}}$ is a scale factor computed from Eq. (4).

Thus, the position of the camera in the georeferenced 
coordinate system $\boldsymbol{T}_{\mathrm{ms}}$ is

$$
\boldsymbol{T}_{\mathrm{ms}}=-\boldsymbol{R}^{\prime} \boldsymbol{t}
$$

According to the installation relation $\boldsymbol{T}_{\mathrm{si}}$ between the OOAS and the lander, the landing trajectory of the lander in the georeferenced coordinate system $\boldsymbol{X}$ is obtained as Eq. (6):

$$
X=T_{\mathrm{ms}} T_{\mathrm{si}}
$$

The reconstructed trajectory is shown in Fig. 12, and Fig. 13 shows the variation in lander altitude with time.

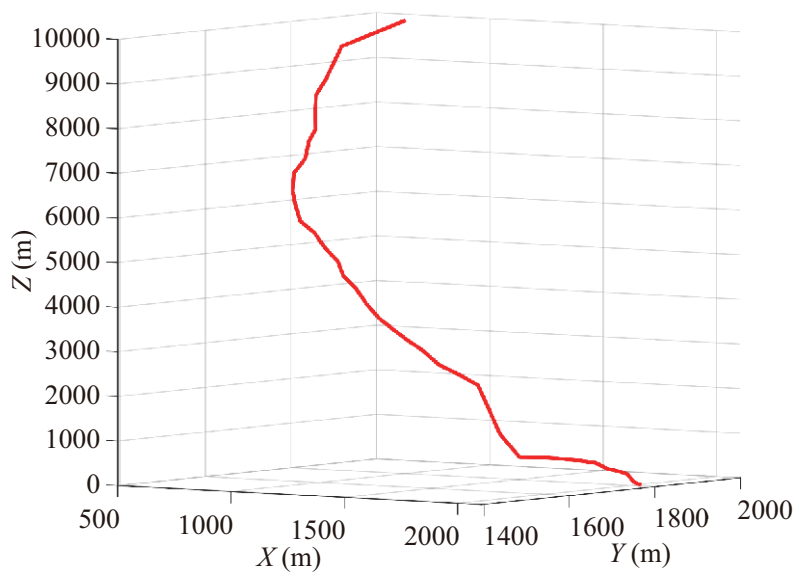

Fig. 12 Reconstructed lander trajectory.

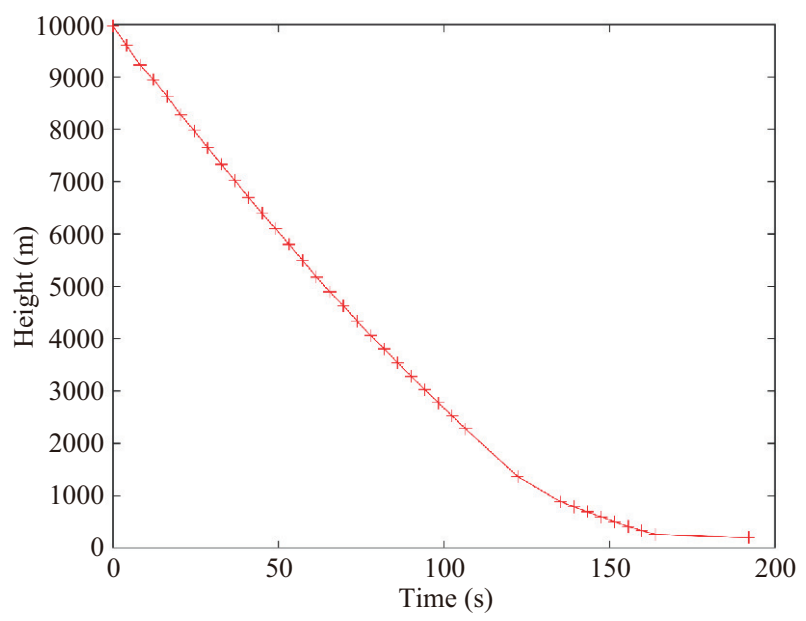

Fig. 13 Variation of lander altitude with time.

The obtained trajectory was projected onto the DOM in the range of $109^{\circ} 54^{\prime} \mathrm{E}-109^{\circ} 56^{\prime} \mathrm{E}$ and $25^{\circ} 3^{\prime} \mathrm{N}-25^{\circ} 4^{\prime} \mathrm{N}$, as shown in Fig. 14. The yellow and green crosses mark the landing site and backshell landing point, respectively. Combined with the reconstructed trajectory in Fig. 12, the descent procedure can be described as follows. First, when descending from an altitude of 10023.79

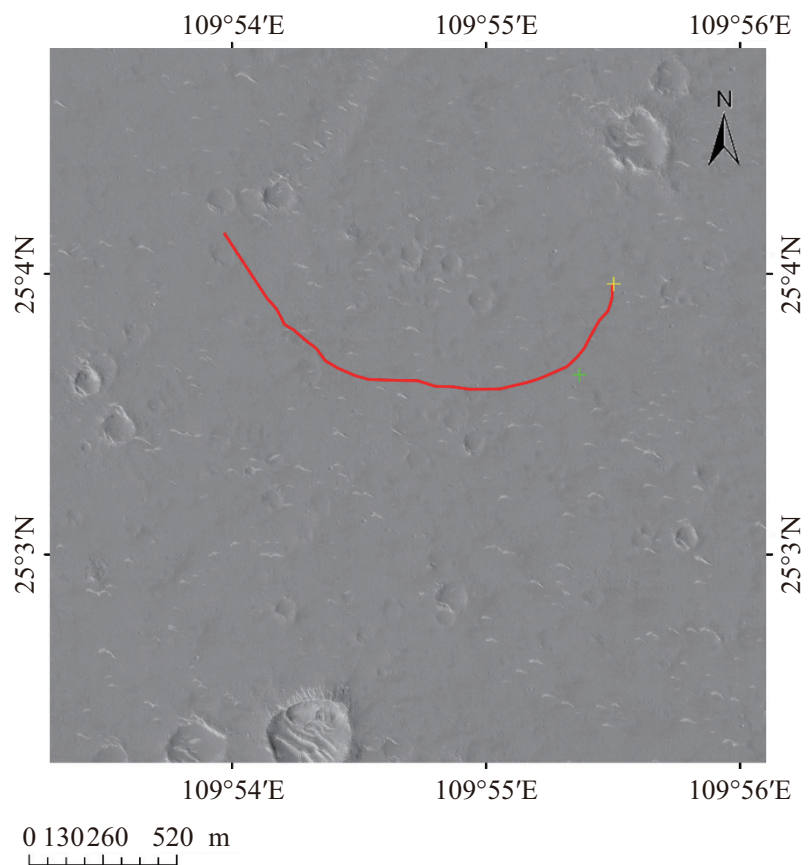

Fig. 14 Projection of the reconstructed trajectory on the DOM.

to $6083.48 \mathrm{~m}$ above the Martian surface, TW-1 moved $1200.00 \mathrm{~m}$ to the southeast. Subsequently, TW-1 adjusted its direction to the east and continued to fly for 1216.50 $\mathrm{m}$. When the lander altitude decreased to $1353.26 \mathrm{~m}$, the backshell was ejected and backshell-avoidance algorithm executed. To avoid the ejected backshell, TW-1 moved $285.00 \mathrm{~m}$ to the northeast between the altitudes of 1353.26 and $100.00 \mathrm{~m}$ and entered the hover and precise obstacle-avoidance phase. At an altitude of $100.00 \mathrm{~m}$, TW-1 hovered and selected a safe landing site. Then, the lander moved to the northeast and crossed a crater with a diameter of $18.00 \mathrm{~m}$, during which the altitude decreased to $69.57 \mathrm{~m}$. Finally, TW-1 descended slowly and landed safely. As seen in Fig. 14, the horizontal distance between the landing points of the lander and backshell was $350.00 \mathrm{~m}$ and the corresponding distance obtained by the navigation camera was $350.82 \mathrm{~m}$ [21]. This shows that the landing trajectory was obtained with an acceptable precision.

To verify the accuracy of the determined trajectory quantitatively, the RMS of the reprojection error and the horizontal error of the ground control points (GCPs) were calculated [22]. In this study, 19 ground control points (GCPs) near the landing site were manually selected on the DOM and OOAS images according to the principle that the preferred choices are smaller craters and rock 
centers and those distributed evenly in the landing area. The distributions of the GCPs in this study are plotted in Fig. 15. With the geometric transformations calculated in Section 3, the GCPs in the DOM were projected onto the OOAS images, and the corresponding projected positions in the OOAS images are regarded as estimation values. The GCPs manually selected in the OOAS images were considered as the true values. The difference between the true and estimated values corresponds to the reprojection error. The horizontal error of the GCPs is the result of multiplying the OOAS image resolution with the

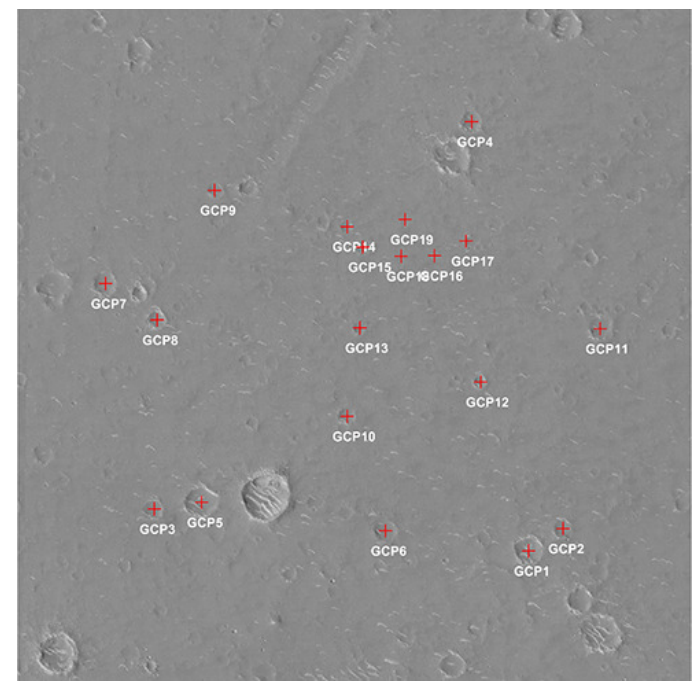

Fig. 15 Distribution of the selected control points in DOM.

Table 2 Deviations of the GCPs

\begin{tabular}{ccc}
\hline GCP & $\begin{array}{c}\text { RMS of reprojection } \\
\text { error (pixel) }\end{array}$ & $\begin{array}{c}\text { Horizontal } \\
\text { error }(\mathrm{m})\end{array}$ \\
\hline GCP1 & 1.08 & 1.63 \\
GCP2 & 1.11 & 1.65 \\
GCP3 & 1.67 & 2.31 \\
GCP4 & 1.26 & 1.74 \\
GCP5 & 1.03 & 1.61 \\
GCP6 & 1.16 & 1.81 \\
GCP7 & 1.21 & 1.40 \\
GCP8 & 1.15 & 1.16 \\
GCP9 & 1.49 & 1.74 \\
GCP10 & 1.60 & 1.82 \\
GCP11 & 1.72 \\
GCP12 & 1.45 & 2.04 \\
GCP13 & 1.76 & 2.35 \\
GCP14 & 1.81 & 1.75 \\
GCP15 & 1.51 & 2.50 \\
GCP16 & 2.07 & 1.53 \\
GCP17 & 1.39 & 1.38 \\
GCP18 & 1.12 & 2.12 \\
GCP19 & 1.66 & 1.80 \\
Global RMS & 1.51 & 1.79 \\
\hline & 1.42 &
\end{tabular}

reprojection error. The RMS values of the reprojection errors and the horizontal errors of the GCPs are presented in Table 2. From Table 2, we can see that the reprojection error of the control points is 1.42 pixels, and the horizontal error is $1.79 \mathrm{~m}$, which is considered to be the accuracy of the TW-1 descent trajectory determination.

\section{Conclusions}

In this study, a hybrid-matching approach was employed to precisely locate the landing site and reconstruct the trajectory of TW-1 using sequential images captured by its OOAS. First, the precise location of the TW-1 landing site was determined to be $109.9259^{\circ} \mathrm{E}, 25.0659^{\circ} \mathrm{N}$ within an accuracy of $1.56 \mathrm{~m}$. This can serve as geographic data support for subsequent Mars-exploration missions. Furthermore, the landing trajectory of TW-1 was reconstructed, and the horizontal error of the trajectory was verified by the selected control points as being $1.79 \mathrm{~m}$. The reconstructed trajectory can be applied to analyze and optimize obstacle-avoidance and autonomous-control strategies. In the future, with the release of higher quality images and greater scientific data, more automatic methods can be utilized to obtain more precise results with increased efficiency.

\section{Appendix OOAS images used in this study}

\begin{tabular}{|c|c|c|}
\hline No. & Image number of OOAS & $\begin{array}{l}\text { Shooting } \\
\text { time }(\mathrm{s})\end{array}$ \\
\hline 1 & 009_000002_20210601212458201-LTO2 & 169456463.738 \\
\hline 2 & 009_000004_20210601212502139-LTO2 & 169456467.834 \\
\hline 3 & $009 \_000005 \_20210601212504085-L T O 2$ & 169456471.930 \\
\hline 4 & $\begin{array}{lll}009 & 000007 & 20210601212508195-L T O 2\end{array}$ & 169456476.026 \\
\hline 5 & $009 \_000009 \_20210601212512362-L T O 2$ & 169456480.122 \\
\hline 6 & 009_000012_20210601212518584-LTO2 & 169456484.218 \\
\hline 7 & 009_000014_20210601212522966-LTO2 & 169456488.314 \\
\hline 8 & $009 \_000015 \_20210601212525392-L T O 2$ & 169456492.410 \\
\hline 9 & 009_000018_20210601212532396-LTO2 & 169456496.506 \\
\hline 10 & 009_000020_20210601212543001-LTO2 & 169456500.602 \\
\hline 11 & 009_000022_20210601212548883-LTO2 & 169456504.698 \\
\hline 12 & 009_000024_20210601212553670-LTO2 & 169456508.794 \\
\hline 13 & 009_000025_20210601212556049-LTO2 & 169456512.890 \\
\hline 14 & 009_000028_20210601212603187-LTO2 & 169456516.986 \\
\hline 15 & $009 \_000029 \_20210601212605622-L T O 2$ & 169456521.082 \\
\hline 16 & 009_000032_20210601212612885-LTO2 & 169456525.178 \\
\hline 17 & 009_000033_20210601212615234-LTO2 & 169456529.274 \\
\hline 18 & 009_000035_20210601213341321-LTO2 & 169456533.370 \\
\hline 19 & 009_000037_20210601213346076-LTO2 & 169456537.466 \\
\hline 20 & 009_000039_20210601213351101-LTO2 & 169456541.562 \\
\hline 21 & 009_000042_20210601213358280-LTO2 & 169456545.658 \\
\hline
\end{tabular}


(Continued)

Shooting

\begin{tabular}{ll} 
No. Image number of OOAS & $\begin{array}{c}\text { Shooting } \\
\text { time }(\mathrm{s})\end{array}$ \\
\hline
\end{tabular}

22 009_000044_20210601213403163-LTO2 169456549.754

$23009-000045 \quad 20210601213405529$-LTO2 169456553.850

$24009-000047$ 20210601213410355-LTO2 169456557.946

25009 -000050_20210601213653172-LTO2 169456562.041

26 009_000051_20210601213420024-LTO2 169456566.137

$27009-000055$ 20210601213436721-LTO2 169456585.971

$28009-000057$ 20210601213441566-LTO2 169456598.907

$29009-000059$ 20210601213446447-LTO2 169456603.003

30 009_000061_20210601213451678-LTO2 169456607.099

31 009_000063_20210601213456631-LTO2 169456611.194

$32009-000065$ 20210601213501360-LTO2 169456615.290

33009 000068_20210601213508778-LTO2 169456619.386

34 009_000069_20210601213511162-LTO2 169456623.482

35009 000071_20210601213515974-LTO2 169456627.578

36 009_000076_20210601213534608-LTO2 169456656.251

\section{Conflict of interest}

The authors declare that there is no conflict of interest.

\section{References}

[1] Wan, W. X., Wang, C., Li, C. L., Wei, Y. China's first mission to Mars. Nature Astronomy, 2020, 4(7): 721.

[2] Li, C. L., Zhang, R. Q., Yu, D. Y., Dong, G. L., Liu, J. J., Geng, Y., Sun, Z. Z., Yan, W., Ren, X., Su, Y., et al. China's Mars exploration mission and science investigation. Space Science Reviews, 2021, 217(4): 57.

[3] Zhao, W. J. Tianwen-1 and China's Mars exploration program. National Science Review, 2021, 8(2): nwaa285.

[4] Cao, J. F., Huang, Y., Hu, X. G., Huang, Q., Li, P. J., Chen, M. Determination of impact site of CE-1 using USB and VLBI data. Journal of Astronautics, 2010, 31(7): 1724-1729. (in Chinese)

[5] Edwards, C. D., Bruvold, K. N., Erickson, J. K., Gladden, R. E., Guinn, J. R., Ilott, P. A., Jai, B., Johnston, M. D., Kornfeld, R. P., Martin-Mur, T. J., et al. Telecommunications relay support of the Mars Phoenix Lander mission. In: Proceedings of the IEEE Aerospace Conference, 2010: 1-13.

[6] Li, J. L., Guo, L., Qian, Z. H., Jiang, D. R., Zheng, W. M., Hong, X. Y. Determination of the controlled landing trajectory of Chang'E-1 satellite and the coordinate analysis of the landing point on the Moon. Chinese Science Bulletin, 2010, 55(13): 1240-1245.

[7] Liu, J. J., Ren, X., Yan, W., Li, C. L., Zhang, H., Jia, Y., Zeng, X. G., Chen, W. L., Gao, X. Y., Liu, D. W., et al. Descent trajectory reconstruction and landing site positioning of Chang'E-4 on the lunar farside. Nature Communications, 2019, 10: 4229.

[8] Wang, J., Wu, W. R., Li, J., Di, K. C., Wan, W. H.,
Xie, J. F., Peng, M., Wang, B. F., Liu, B., Jia, M. N., et al. Vision based Chang'E-4 landing point localization. Scientia Sinica Technologica, 2020, 50(1): 41-53.

[9] Liu, B., Xu, B., Liu, Z. Q., Liu, Y. L., Di, K. C., Tang, G. S., Zhou, J. L. Descending and landing trajectory recovery of Chang'e-3 lander using descent images. Journal of Remote Sensing, 2014, 18(5): 981-987. (in Chinese)

[10] Zou, Y. L., Zhu, Y., Bai, Y. F., Wang, L. G., Jia, Y. Z., Shen, W. H., Fan, Y., Liu, Y., Wang, C., Zhang, A. B., et al. Scientific objectives and payloads of Tianwen-1, China's first Mars exploration mission. Advances in Space Research, 2021, 67(2): 812-823.

[11] Meng, Q. Y., Wang, D., Wang, X. D., Li, W., Yang, X. W., Yan, D. J., Li, Y., Cao, Z. R., Ji, Q., Sun, T., et al. High resolution imaging camera (HiRIC) on China's first Mars exploration Tianwen-1 mission. Space Science Reviews, 2021, 217(3): 42.

[12] China National Space Administration. Tianwen-1 high resolution imaging camera dataset. Lunar and Planetary Data Release System, 2020. Available at http://moon. bao.ac.cn.

[13] Maass, B., Woicke, S., Oliveira, W. M., Razgus, B., Krüger, H. Crater navigation system for autonomous precision landing on the moon. Journal of Guidance, Control, and Dynamics, 2020, 43(8): 1414-1431.

[14] He, J., Cui, H. T., Feng, J. H. Edge information based crater detection and matching for lunar exploration. In: Proceedings of the International Conference on Intelligent Control and Information Processing, 2010: 302-307.

[15] Johnson, A. E., Montgomery, J. F. Overview of terrain relative navigation approaches for precise lunar landing. In: Proceedings of the IEEE Aerospace Conference, 2008: $1-10$.

[16] Park, W., Jung, Y., Bang, H., Ahn, J. Robust crater triangle matching algorithm for planetary landing navigation. Journal of Guidance, Control, and Dynamics, 2019, 42(2): 402-410.

[17] Hanak, C., Crain, T., Bishop, R. Crater identification algorithm for the lost in low lunar orbit scenario. $A d$ vances in the Astronautical Sciences, 2010, 137(32): 2010.

[18] Mourikis, A. I., Trawny, N., Roumeliotis, S. I., Johnson, A. E., Ansar, A., Matthies, L. Vision-aided inertial navigation for spacecraft entry, descent, and landing. IEEE Transactions on Robotics, 2009, 25(2): 264-280.

[19] Johnson, A. E., Cheng, Y., Montgomery, J., Trawny, N., Tweddle, B. E., Zheng, J. Design and analysis of map relative localization for access to hazardous landing sites on Mars. In: Proceedings of the AIAA Guidance, Navigation, and Control Conference, 2016: 
AIAA 2016-0379.

[20] Information on http://www.cnsa.gov.cn/n6758823/n67 58838/c6812111/content.html (cited 20 Aug 2021).

[21] Liang, X., Chen, W. L., Cao, Z. X., Wu, F. Z., Lyu, W. Z., Song, Y. Z., Li, D. F., Yu, C. W., Zhang, L., Wang, L. The navigation and terrain cameras on the Tianwen-1 Mars rover. Space Science Reviews, 2021, 217(3): 1-20.

[22] Liu, J. J., Yan, W., Li, C. L., Tan, X., Ren, X., Mu, L. L. Reconstructing the landing trajectory of the CE-3 lunar probe by using images from the landing camera. Research in Astronomy and Astrophysics, 2014, 14(12): 1530-1542.

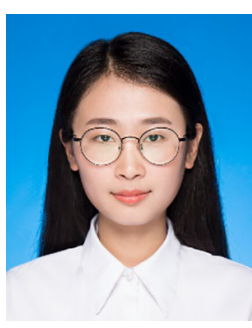

Anran Wang received her M.S. degree from the University of Information Engineering in 2019, and now she is a Ph.D. candidate at Beijing Institute of Control Engineering, mainly engaged in research on computer vision, visual navigation and localization. E-mail: war07101@163.com.

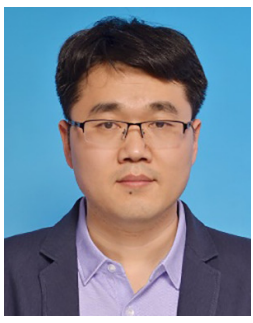

Li Wang received his B.S., M.S., and Ph.D. degrees from Northwestern Polytechnical University in 1998, 2001, 2004, respectively, mainly engaged in research on spatial optoelectronic technology and machine vision. E-mail: wupeng3992@163.com.

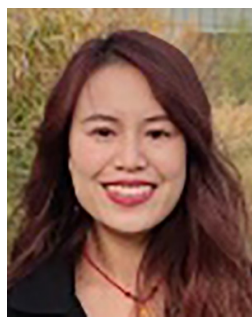

Yinuo Zhang received her B.S. degree from Wuhan University in 2016 and her M.S. degree from Wuhan University in 2018, mainly engaged in remote sensing applications, image processing algorithms, etc. E-mail: ZhangYn950303@163.com.

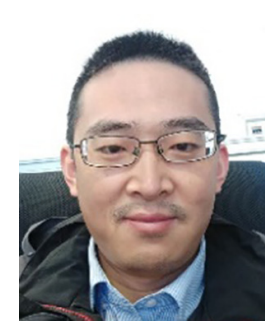

Baocheng Hua received his B.S. degree from North University of Technology in 2005 and Ph.D. degree from Beihang University in 2011, mainly engaged in the research of machine vision, optical navigation, deep space exploration, etc. E-mail: 85340287@qq.com.

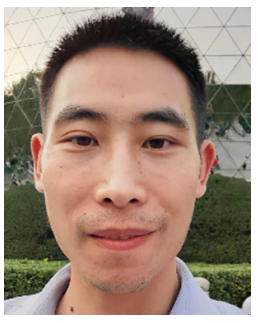

163.com.
Tao Li received his bachelor degree from Zhengzhou University in 2007, master degree from Beijing Institute of Technology in 2009, and Ph.D. degree from Beijing Institute of Technology in 2014, mainly engaged in digital image processing, visual measurement, structured light, etc. E-mail: ietaoli@

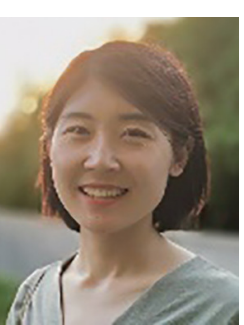

Yang Liu received her B.S. degree from Nanjing University of Aeronautics and Astronautics in 2015 and her M.S. degree from China Academy of Space Technology in 2018, mainly engaged in image processing, landing obstacle avoidance, etc. E-mail: ly15605188503@163.com.

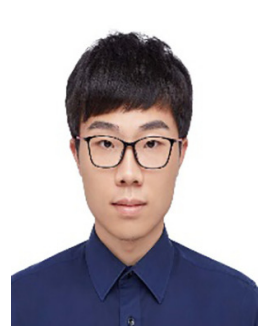

Danyong Lin received his master degree from Beijing Institute of Technology in 2019, and is now a Ph.D. student in Beijing Institute of Control Engineering, mainly engaged in computer vision, 3D points cloud reconstruction. E-mail: 13001044577@163.com.

Open Access This article is licensed under a Creative Commons Attribution 4.0 International License, which permits use, sharing, adaptation, distribution and reproduction in any medium or format, as long as you give appropriate credit to the original author(s) and the source, provide a link to the Creative Commons licence, and indicate if changes were made.

The images or other third party material in this article are included in the article's Creative Commons licence, unless indicated otherwise in a credit line to the material. If material is not included in the article's Creative Commons licence and your intended use is not permitted by statutory regulation or exceeds the permitted use, you will need to obtain permission directly from the copyright holder.

To view a copy of this licence, visit http://creativecomm ons.org/licenses/by/4.0/. 